\title{
LAS ELECCIONES GENERALES DE ABRIL DE 1992 EN ITALIA ${ }^{1}$
}

\author{
José Girón \\ Jorge Torre \\ Universidad de Oviedo
}

\section{INTRODUCCION}

Durante el último año y medio Italia ha sido noticia diaria en todos los medios de comunicación. Lo que empezó siendo un problema de comisiones ilegales en la ciudad de Milán, se convirtió en un escándalo nacional al descubrirse la irregular financiación de la mayoría de los partidos políticos y las relaciones entre política y mafia. Tras dieciocho meses de investigaciones estan abiertos 1.200 sumarios judiciales y casi 500 personas han sufrido prisión preventiva. En los últimos meses han tenido que dimitir cuatro secretarios generales de partidos acosados por la magistratura, y líderes emblemáticos como Bettino Craxi y Giulio Andreotti. Ante esta situación de completa crisis del sistema político se necesita una profunda regeneración. Algunos analistas comentan que Italia esta atravesando actualmente la transición de la I a la II República. El referéndum del 18 de abril de 1993 en el que un $82,9 \%$ del electorado ha votado a favor de cambiar el sistema electoral proporcional por el mayoritario para la elección del Senado y el $90,3 \%$ a favor de modificar la financiación pública de los partidos, parece iniciar ese proceso. Es interesante constatar la desconfianza del electorado sobre la partitocracia histórica y un apoyo significativo a un partido nuevo. sin lastres. la Liga Norte-Liga Lombarda. En las siguientes páginas trataremos de analizar, sin ánimo exhustivo, las elecciones generales celebradas en la República de Italia, los días 5 y 6 de abril de 1992. realizando un comentario de la ley electoral, de la situación política previa al proceso electoral, una breve historia de los principales partidos políticos que concurrieron a los comicios, un resumen de los programas, la campaña y los resultados electorales.

\section{LA LEY ELECTORAL}

Italia tenía un sistema electoral mixto de representación proporcional por lista con cociente rectificado, restos más amplios y voto preferente ${ }^{2}$. Este sistema convierte a Italia en uno de los países del mundo con mayor grado de representatividad.

\footnotetext{
I Los autores agradecen a los profesores (iian Primo Cella y Alfonso Botti la lectura y comentarios al presente articulo.

2 W.J.M. MACKENZII:, Elecciones libres. Tecnos, Madrid, 1962, pp. 100-103; y Jean Marie COTTERET y Claude EMIRI, Los sistemas electorales, Oikos-Tau, Barcelona, 1973, pp. 92 y 103.
} 
Comentaremos brevemente la ley electoral para conocer el marco jurídico en el que se desarrollan las elecciones ${ }^{3}$. La Cámara de Diputados se compone de 630 miembros y el voto es obligatorio. Existe una sola papeleta, donde figuran todos los partidos -a través de sus símbolos- y sobre la cual el elector debe señalar el anagrama del partido elegido y escribir a su derecha el apellido del candidato seleccionado de la lista ofrecida por el partido.

En estas elecciones se han introducido algunas modificaciones con respecto a consultas anteriores. La legislación electoral básica de 1952, la Ley Scelba, fue modificada en 1957, y retocada por la ley de 21 de marzo de 1990, para garantizar una mayor eficiencia y transpariencia en el proceso electoral. Entre otros temas, queda prohibido realizar el escrutinio de los votos de los partidos separadamente de los votos de preferencia nominal. Por vez primera aparece el color en los símbolos utilizados por los partidos en la papeleta, con la finalidad de facilitar la identificación a los electores, ante la proliferación de listas con emblemas semejantes. También por primera vez la colocación de los partidos en la papeleta se designa mediante un sorteo en cada circunscripción.

El referéndum sobre la "preferencia única" celebrado el 7 de junio de 1991 introdujo una novedad en el sistema electoral. Hasta entonces, los electores podían expresar en la papeleta hasta un total de cuatro preferencias, a partir de ahora, sólo se puede incluir una. Además ésta no podrá ser expresada con el número de la lista sino que se tiene que escribir el apellido del candidato.

Como no existe voto por correo, el Estado realiza unos amplios descuentos sobre el precio de los transportes publicos $-30 \%$ en avión, $60 \%$ en tren, $100 \%$ en barcopara que los electores se trasladen desde sus lugares de residencia -incluso desde el extranjero- a los colegios electorales donde esten censados.

Otra diferencia con respecto a España, es el tiempo en el que se realiza la elección, que transcurre durante dos jornadas. De las siete de la mañana a las diez de la noche del domingo, y de las siete a las dos del mediodía del lunes. Esta situación facilita y explica la extraordinaria afluencia del electorado a las urnas, si bien, hay que hacer también mención a la circunstancia de que la ciudadanía italiana considera el voto como un deber cívico además de un derecho ${ }^{4}$. Por todo ello, en Italia se alcanzan unos porcentajes de participación de los más altos de Europa, sólo superados por Holanda y Austria.

\section{LA INTROMISION DEL JEFE DEL ESTADO EN LA POLITI- CA ITALIANA}

Una peculiaridad de la política italiana, en los últimos tres años, fue la constante

\footnotetext{
${ }^{3}$ Le Leggi Elettorali, Ministero dell'Intemo, Roma, 1992, 272 págs.

${ }^{4}$ Giacomo SANI, "Ciudadanos y sistema politicu: participación y cultura politica de masas en lialia", en Revista de Estudios Politicos, n⿳ 79, enero-marzo 1993, pag. 136.
} 
injerencia del Jefe del Estado. Francesco Cossiga, en la política cotidiana, a través de sus declaraciones sobre diversos temas de actualidad, lo que provocó reacciones en cadena de los partidos políticos. Aún más polémica fue su interferencia continua con los otros poderes del Estado. En el otoño de 1991, cuando el tema Gladio acaparaba la atención nacional. Cossiga no sólo trató de impedir la formación de una comisión parlamentaria sino que también justificó la existencia de Gladio como una respuesta a la "agresión comunista" en plena guerra fría.

Sus tomas de postura, ante diferentes temas, introducen un elemento de tensión en la vida política. Así, se produjo un duro enfrentamiento entre Cossiga y la Magistratura, al tratar de obstaculizar la labor de la justicia en el tema Gladio, hasta el punto de amenazar con la intervención de los Carabineros para desalojar a los jueces reunidos en sesión del Consejo Superior de la Magistratura. La situación creada llevó a un enfrentamiento, sin precedentes, del Poder Judicial -con huelga incluida- con la Jefatura del Estado. Simultáneamente, una parte de las Fuerzas Armadas -los Carabinieri- no permanecieron ajenas a la situación, decantándose hacia la posición de Cossiga, lo que llevó a numerosos comentaristas políticos a hablar de la amenaza de un golpe de Estados.

Días antes de los acontecimentos narrados y ante la situación de incalificable intromisión de Cossiga en la vida política, lo que, por otra parte, constituye -según numerosos juristas- un atentado a la Constitución, el PDS estudiaba la posibilidad de actuar legalmente contra el Jefe del Estado ${ }^{6}$. En los mismos días de la polémica con la Magistratura, el PDS presentó en la Cámara de Diputados un procedimiento de acusación contra Cossiga, es decir, un "impeachment", si bien no recibió el apoyo de otras fuerzas políticas y el intento fracaso ${ }^{7}$.

Coincidiendo con la disolución del Parlamento, la prensa se hizo eco de otro polémico tema. Durante sus investigaciones sobre la figura de Togliatti y el Komintern, un profesor de historia contemporánea de la Universidad de Pisa, hizo pública una información, hasta entonces desconocida, según la cual el prestigioso líder comunista quedaba en entredicho. La historia sería la siguiente: durante la II Guerra Mundial, Mussolini envió a varias decenas de miles de italianos a combatir contra la URSS, varios miles de ellos fueron hechos prisioneros por los soviéticos y parece ser que Togliati -numero tres de la Komintern- envió una carta a Vicenzo Bianco, delegado del PCI ante la III Internacional, en la que demostraría su desprecio por la suerte de sus compatriotas ${ }^{8}$. Una información de este tipo perjudicaba evidentemente las espectativas de voto del PDS, justo antes de la convocatoria de las

5 La Repubblica, 5 y 6 de diciembre de $\mid 991$.

${ }^{6}$ La Repubblica, 4 de diciembre de 1991.

${ }^{7}$ La Repubblica, 25 enero y 6 febrero de 1992.

${ }^{8}$ El presunto texto decia "se un buon numero di prigionieri muore in conseguenza delle dure condizioni di fatto non ci trovo assolutamente niente da dire. Sarà una lezione per l'Italia...". La Repubblica, 2 de febrero de 1992. El ejercito italiano enviado a luchar a la URRS estaba formado por 229.000 hombres, de los cuales solamente regresaron 10.100, a comienzos de 1946. La Repubblica, 9 de febrero de 1992. 
elecciones generales.

Francesco Cossiga no desaprovechó la ocasión de atacar al PDS $^{9}$ y por extensión a Nilde Iotti, esposa de Togliatti, presidenta de la Cámara de Diputados y probable candidata del PDS a la sucesión de aquél, en la próxima renovación en la Jefatura del Estado ${ }^{10}$. Convencido -interesadamente- de la correcta interpretación realizada, propuso una comisión de investigación que se trasladase a Moscú para verificar el tema. Prestigiosos intelectuales y políticos de varios partidos reconocieron que las palabras de Togliatti se habían sacado de contexto y que una carta escrita hacía casi medio siglo no debía influir en la política actual. El propio secretario general de la Democracia Cristiana, Arnaldo Forlani, consideró que "no queremos especular con el pasado comunista", justo en un momento en el que en otros medios informativos se había publicado una carta de De Gasperi, fundador de la Democracia Cristiana, quien había demostrado su "complacencia" ante la invasión nazi de la URSS". Finalmente, el tema fue archivado y el propio Cossiga se vio obligado a reconocer su derrota en el asunto ${ }^{12}$.

\section{LOS PARTIDOS POLITICOS Y LOS PROGRAMAS ELECTO- RALES}

La alta proporcionalidad del sistema electoral ha conducido a la formación de un sistema de partidos del tipo "pluralismo extremo, multipolar y centrífugo" ${ }^{13}$. Desde las primeras elecciones de la postguerra -1948- hasta la actualidad, nueve fuerzas políticas de ámbito nacional tienen asegurada su representación en la Cámara. A estos partidos históricos hay que sumar varios aparecidos en los últimos años que junto a otros regionales y locales, totalizan 247 listas presentadas en estas elecciones $^{14}$. A continuación haremos referencia a las 12 principales fuerzas políticas presentes en el proceso clectoral. El esquema empleado es igual para cada una de ellas: un mínimo apunte histórico, un comentario de su actual situación política y gubernamental, un breve resumen de los principales puntos de su programa electoral y los lemas empleados durante el proceso electoral.

\footnotetext{
${ }^{9}$ La Repubblica, 6 de febrero de 1992.

${ }^{10}$ La Repubblica, 27 de febrero de 1992.

"La Repubblica. 9 de febrero de 1992.

${ }^{12}$ La Repubblica, 9 de febrero de 1992. Cossiga reconoció "Togliatti. ecco chi mi a sconfitto". Además, poco despues, se hizo publica la version original de la supuesta carta de Togliatti y se demostró la manipulación de la carta por parte del profesor Franco Andreucci.

${ }^{13}$ Dieter N(HILFN. Sistemas clectorales del mundo, Centro de Estudios Constitucionales, Madrid. 1981, pag. 479.

${ }^{14}$ La Repubblica. 24 de febrero de 1992.
} 


\section{Democracia Cristiana}

Sucesor del Partido Popular -fundado en 1919 por Luigi Sturzo y disuelto en 1926-, algunos de sus cuadros, junto a otros grupos católicos, constituyen entre 1942 y 1943 la DC, bajo el liderazgo de Alcide De Gasperi ${ }^{15}$. El partido se concibe como interclasista, basado en la moral catolica y con corrientes internas. De Gasperi convertirá a la DC en una eficaz maquina electoral, capaz de permanecer desde 1948 hasta hoy día ininterrumpidamente en el gobierno. El partido aunque apoyado por el Vaticano se mantiene. en la medida de lo posible. distante del clericalismo ${ }^{16}$. En 1959 se crea una importante corriente conocida como los "doroteos" liderada por Colombo, Taviani y Moro ${ }^{17}$. Este último, jefe de gobierno entre 1963-68, y una de las grandes figuras de la política de los años sesenta y setenta, abrió el gobierno de coalición hacia la izquierda dando entrada a los socialistas. Las grandes protestas sociales de 1968-73 obligan a la DC a aceptar reformas: estructura del Estado regional, Estatuto de los trabajadores y Ley del divorcio, entre otras. Se produce una renovación en la secretaría general del partido, sale Fanfani y entra Zaccagnini, apoyado por los "renovadores" liderados por Aldo Moro ${ }^{18}$. El secuestro y asesinato de Moro por las Brigadas Rojas en mayo de 1978 produce otra grave crisis en la $\mathrm{DC}^{19}$. Los años ochenta se caracterizan por el liderazgo de Andreotti, con Arnaldo Forlani en la secretaría general (1980-82 y 1989-92) y por la alianza con el PSI de Craxi. En los ultimos años la DC muestra signos de debilidad que se caracterizan por una perdida de la confianza electoral y la salida del partido de jovenes líderes como Leoluca Orlando o el disenso de Mario Segni. En el partido continuan conviviendo corrientes muy heterogéneas, desde una derecha conservadora (Andreotti). pasando por el centro (Forlani, actual secretario general) y una izquierda progresista ( De Mita). Se considera el eje sobre el que pivota toda la política italiana, y se puede identificar con la propia estructura del Estado. Esto lleva a que los deseos de algunas fuerzas políticas y sociales de renovar las instituciones se conviertan en ataques a la propia DC.

Programa. Reforma electoral: premio de 75 escaños a la lista mayoritaria, con la finalidad de crear mayorías estables. Mayor autonomía legislativa y financiera para las regiones. Privatizaciones y crecimiento de las retribuciones igual a la tasa

\footnotetext{
${ }^{15}$ Giuseppe CHIARANTE. La Democrazia Cristiana, Riuniti, Roma, 1980, pag. 13-14 y 18-22.

${ }^{16}$ Maurizio PIERETTI. "De Gasperi c la Democrazia Cristiana", en VV.AA. Storia della societá italiana, vol. XXIII, Teti, Milano, 1982, pag. 81.

${ }^{17}$ G. CANDELORO. Storia dell'/talia moderna, vol. XI, Feltrinelli, Milano, pag. 329.

${ }^{18}$ Entre los "renovadores" estaban tambien Mariano Rumor y Ciriaco De Mita. Los "moderados" estaban liderados por Giulio Andreotti. EI XIII Congreso del partido celebrado en Roma en 1976 fue uno de los mas tormentosos de la historia de la IXC, y en el mismo, los "renovadores" obtuvieron la victoria por un estrecho margen. Cfr. Paul (ilNSBORG, Storia d'ltalia dal dopoguerra a oggi, Einaudi, Torino, 1989 , pag. 502.

${ }^{19} \mathrm{El}$ asesinato de Aldo Moro continua hoy dia siendo motivo de fuere controversia y sobre todo la actuacion de Andreotti en el tema. Sin duda. la desaparicion de Moro beneficio a la corriente mas conservadora de la DC, capitaneada precisamente por Andreotti.
} 
programada de la inflación. Convergencia con Europa reduciendo el déficit y la inflación. Decisiva lucha contra el crimen organizado, sin recurrir a leyes excepcionales. Defensa de la familia como base de la sociedad. Política de seguridad y defensa de la paz en el marco de la OTAN ${ }^{20}$. Lema: "Primero ltalia", "Haz vencer tu futuro", "Quieren disgregar Italia, juntos lo impediremos". Emblema: un escudo cruzado con la palabra "Libertas" en rojo y blanco.

\section{Partido Socialista Italiano}

Fundado en 1892 por Filippo Turati, fue el primer partido de masas de la historia de Italia $^{21}$. Desde los comienzos se hicieron presentes los conflictos entre los reformistas liderados por Turati y los revolucionarios de Labriola ${ }^{22}$. El partido se consolida en los primeros años del siglo y alcanza una gran implantación en el movimiento obrero gracias a su organización y al sindicato Confederazione Generale del Lavoro (CGL) que en 1908 tenía 217.000 miembros $^{23}$. Tras la primera guerra mundial consigue un gran exito electoral en 1919 con el 32,3\% de los votos y 156 diputados $^{24}$. En el Congreso de Livorno (1921) se produce la ruptura entre reformistas y comunistas. En agosto de 1943 se crea el Partido Socialista Italiano de Unidad Proletaria (PSIUP) dirigido por Pietro Nenni que se declara heredero del viejo PSI. Participa en los gobiernos de unidad nacional y en las elecciones de 1946 es el partido de izquierda más votado y el segundo del país tras la DC, con el 20,7\% de los votos ${ }^{25}$. La estrategia del PSIUP sera la alianza con el PCI -lo que provoca la escisión del ala socialdemocrata- y el partido regresa a su denominacion original (PSI). A partir de 1956 abandona la alianza con los comunistas e inicia un acercamiento hacia la DC que se concreta, en 1963, con la entrada en el gobierno de Aldo Moro, con Nenni en la vicepresidencia. Se inicia así una larga etapa en la que el PSI participa en los sucesivos gobiernos de coalición junto con la DC y otros partidos menores. Desde los años setenta con la progresiva polarización del voto entre DC y PCI, el PSI busca un espacio político entre ambos de la mano de Betttino Craxi, secretario general desde 1976. El fuerte liderazgo de Craxi dota al partido de mayor pragmatismo y lo saca de una fase aletargada durante los años sesenta y setenta, para conducirlo hacia posiciones de mayor influencia en la vida política.

\footnotetext{
20 Il Popolo, 1 y 2 de abril de 1992, pp. 16-21 y 13-19 respectivamente.

${ }^{21}$ Giampiero CAROCCI, Giolitti e l'età giolittiana, Einaudi, Torino, 1971, pags. 64-79.

${ }^{22}$ Maurizio RIDOLFI, II PSI e la nascita del partito di massa (1892-1922), Laterza, Bari, 1992, pag. 158.

${ }^{23}$ Angelo MOLAIOLI, PSI. Novanta anni di storia (1892-1982), Istituto Socialista di Studi Storici, Roma, 1982, pag. 109.

24 Pier Luigi BALLINI, Le elezioni nella storia d'Italia dall Unità al fascismo, il Mulino, Bologna, 1988, pp. 189-193.

${ }^{25}$ Piergiorgio CORBETTA. Arturo PARISI y Hans SCHADEE. Elezioni in Italia, il Mulino, Bologna, 1988, pag. 511. Esta fue la unica ocasion en toda la historia de la Republica italiana en la que el partido Socialista obtuvo una votacion superior al partido Comunista.
} 
Entre 1983 y 1987, Craxi desempeñó la presidencia del Consejo de Ministros y, sin entrar en su criticada gestión, lo cierto es que fueron cuatro años de estabilidad gubernamental, sin precedentes en los últimos tiempos. Su proximidad al aparato del poder, han convertido al PSI en un partido también identificado con el Estado, lo que le lleva a recibir fuertes ataques de otras formaciones políticas renovadoras.

Programa. Reforma electoral: a favor de mantener el sistema proprocional actual, si bien introducciendo la barrera del 5\% para eliminar a los pequeños partidos. Elección directa del Jefe del Estado. Moderación salarial para luchar contra la inflacción. Nueva política industrial y agrícola. Reforma de la Magistratura. Necesidad de un Estado social justo y eficiente. Reforma de la enseñanza media (escuela obligatoria hasta los 16 años). Nueva organización de las Fuerzas Armadas dedicadas sólo a la defensa nacional. Y una política de ayuda al desarrollo económico y político de Albania, territorio históricamente ligado a Ios intereses italianos $^{26}$. Lema: "Un gobierno para la recuperación". Emblema: Un círculo rojo con la frase "PSI. Unidad Socialista", y en el centro un clavel rojo sobre fondo blanco.

\section{Partido Democrático de la Izquierda}

Heredero del histórico Partido Comunista Italiano. El PCI nació en el congreso de Livorno de 1921, como escisión del PSI. Tras su tercer congreso, en 1926, fueron sus principales dirigentes y teóricos Gramsci y Togliatti ${ }^{27}$. Durante el fascismo sufre una fuerte represión y será una de las fuerzas basicas de la lucha clandestina. Forma parte de los gobiernos de "unidad nacional" hasta que en 1947 De Gasperi decide la expulsión de los socialistas y comunistas del gobierno. En los años cincuenta consolida su posicion hegemónica en la izquierda ${ }^{28}$. Partido de la clase obrera mantuvo siempre una estrecha relación con la poderosa Confederación General Italiana del Trabajo (CGIL) ${ }^{29}$, sindicato que en los años cincuenta llegó a sobrepasar los tres millones de afiliados ${ }^{30}$. La muerte de Togliatti en 1964 acentuó el enfrentamiento entre el sector de la "derecha" (Amendola y Napolitano) que deseaba una apertura hacia el PSI y el sector de la "izquierda" (Ingrao) que pretendía una alianza de carácter anticapitalista. La derrota de la izquierda abrió el paso a un candidato de "centro", Enrico Berlinguer, que fue elegido secretario general en 1972. Un año

\footnotetext{
${ }^{26}$ Programma Elettorale del PSI. Modena, 1992, 91 pags.

${ }^{27}$ Giorgio AMENDOLA, Storia del PCl (1921-1943), Reuniti, Roma, 1978, pags. 10-42.

${ }^{28}$ Entre 1946 y 1956 el PCI cuenta con un numero de militantes que supera los dos millones. Las cifras mas altas corresponden a 1947 ( 2.252.446) y 1954 (2.145.317). Cfr. Celso GHINI, "Gli iscritti al partito e alla FGCI (1943/1979)". en Massimo ILARDI e Aris ACCORNERO, Il Partido comunista italiano. Struttura e storia dell organizzazione 192111979, Feltrinelli, Milano, 1982, pag. 237.

${ }^{29}$ Gianni FERRANTE, "Interscambio di dirigenti tra parito e sindacato", en ILARDI e ACCORNERO, op. cit., pag. 673-91.

${ }^{30}$ La CGIL tenía en 1957, 3 millones 119 mil afiliados. Cfr. Vittorio FOA. "Sindacati e classe operaia", en Valerio (ASIRONOVO (a cura di) L'Italia Contemporanea 1945-1975. Einaudi, Torino, 1976, pag. 261.
} 
después, Berlinguer presenta la política del "compromiso histórico" entre los tres principales partidos (DC, PSI y PCI) para evitar cualquier tentación autoritaria y combatir la degradación moral del país mediante la convergencia de la moralidad católica y los valores comunistas ${ }^{31}$. Su estrategia no fructifica tanto por las resistencias de una parte de las sociedad italiana como por las presiones extranjeras. Tras el "compromiso histórico" se intenta la vía de la "alternativa democrática" de alianza con el PSI. Los años ochenta se caracterizan por un estancamiento y una caída electoral (del 34,4\% en 1976 al 26,6\% en 1987) ${ }^{32}$. En 1988 Achille Occhetto llega a la secretaria general. Ante el derrumbamiento del socialismo real en la Europa del Este, Occhetto propone la creación de un nuevo partido. En el XX Congreso del PCI, celebrado en Rimini en 1991, la mayoría de los delegados decidieron retocar el programa, cambiar el viejo nombre y adaptarse a los nuevos tiempos, creando el Partido Democrático de la lzquierda (PDS). El principal impulsor de la renovación fue Achille Occhetto, secretario general del ex-PCI y del nuevo PDS. Este cambio, provoca la escisión de la minoría que forma Refundación Comunista ${ }^{33}$.

Programa. Reforma electoral: sistema mixto, con premio para la lista que superase el $40 \%$ de los votos. Reforma constitucional: Fortalecer las competencias del Ejecutivo, reducción del número de diputados a 400 , transformar el Senado en Cámara de las Regiones con 200 miembros. Autonomía tributaria para las regiones. Política de pleno empleo. Reforma fiscal, reforma de la administración financiera. creación de un impuesto ecológico. Subida de las pensiones, salario mínimo garantizado. Fomento de la enseñanza pública y obligatoria hasta los 16 años $^{34}$. Lema: "El partido de los trabajadores", "Somos la Italia que dice basta". "La oposición que construye". Emblema: un gran roble de color verde que hunde sus raíces en una pequeña bandera roja comunista.

\section{Partido Republicano Italiano}

Fundado a finales del siglo pasado -en 1895-, con un programa que defendía la implantación de la República y una nueva organización del Estado. En sus primeros años fue un partido pequeño ya que su espacio político fue ocupado progresivamente por el partido Socialista ${ }^{35}$. A partir del congreso de Roma de 1908 su implantación empieza a crecer impulsando una política reformista y anticlerical. Durante el fascismo pasa a la clandestinidad y organiza el grupo Giustizia e Libertà. En 1946 una parte del Partito d'Azione confluye en el PRI que se sitúa en el área de gobierno. En 1963 entra en los gobiernos de centro-izquierda presididos por Aldo Moro. En 1981 el republicano Giovanni Spadolini forma el primer gobierno presidido

\footnotetext{
${ }^{31}$ Pietro SCOPPOLA, La repubblica dei partiti, il Mulino, Bologna, 1991, pag. 365-366.

32 CORBETTA. PARISI, SCHIDE. : op. cit., 524-531.

33 L.a mocion de Occhetto obtuvo el $65.9 \%$ de los votos y las contrarias, el $30,0 \%$ la de lngrao y el 3.9\% la de Cossutta. Cfr. Piero ICiNAZI, Dal PCI al PDS, il Mulino. Bologna, 1992. 133.

${ }^{34}$ Suplemento de $L \cdot$ Unità, 3 de abril de 1992, pp. 4-14.

35 (iiorgio (iAI.I,I, I partiti politici in Italia (1861-1973). UTET, Torino, 1975, pag. 130.
} 
por un no democristiano en toda la historia de la República, apoyado por el pentapartido dura 17 meses. En 1987 Giorgio La Malfa es elegido secretario general en sustitución de Spadolini. Dos años después La Malfa impulsa un cambio de orientación en el seno del partido, proponiendo una nueva alternativa basada en el centro y abierta a la izquierda, la llamada "agregación de fuerzas laicas", de donde naturalmente quedaría excluida la DC. Como primer paso hacia el objetivo anterior, en 1991 el PRI abandona el gobierno del pentapartido y pasa a la oposición. El partido tiene actualmente cien mil afiliados. Ante las elecciones, un grupo de conocidos intelectuales y hombres de negocios publicaron una nota en la prensa expresando su apoyo al partido Republicano ${ }^{36}$.

Programa. Renovación del sistema político, basado en una reforma electoral (introduciendo el distrito uninominal que reduzca la fragmentación política y la elección directa de los alcaldes y presidentes de la regiones), y una reforma institucional (gobierno con más poderes, a favor de un gobierno de los "técnicos" no partidarios, y desarrollo del regionalismo). En la economía: privatización de algunos sectores públicos, revisión del sistema fiscal, menor intervención estatal en la economía. Lucha contra la criminalidad y la corrupción ${ }^{37}$. Lema: "Un voto joven y cualificado para cambiar y crecer", "Yo voto por una Italia nueva", "El voto republicano. El desafío de la Italia civil". Emblema: una hoja de hiedra de color verde.

\section{Partido Socialista Democrático Italiano}

En el congreso del partido Socialista celebrado en enero de 1947, el grupo moderado -opuesto a la colaboración con los comunistas- liderado por Giuseppe Saragat se escindio fundando el Partido Socialista de los Trabajadores Italianos ${ }^{38}$. Desde el primer momento se sitúa en el área de gobierno aliado a la DC. En su primer congreso, celebrado en 1948, establece su ideología reformista y decididamente anticomunista. En 1952 pasa a denominarse Partido Social Demócrata Italiano (PSDI). En 1964 Saragat es elegido Presidente de la República. Dos años después el PSDI se unifica con el PSI pero en 1969 sobrevino una nueva ruptura. Las décadas del setenta y ochenta significaron un estancamiento de sus posiciones electorales y progresivamentc fue nutriendo sus votos de las regiones sur del país ${ }^{39}$. Desde los años de su fundación ha participado en el Gobierno, en coalición con la DC, perteneció al pentapartido y al cuatripartido actualmente en el poder. Desde 1988 el secretario general es Antonio Cariglia (opuesto a la unión con el PSI) y cuenta con cien mil afiliados. Se caracteriza por ser un partido pequeño y tranquilo

\footnotetext{
${ }^{36}$ Corriere della Sera, 4 de abril de 1992, pag. 24. Algunos de los 16 firmantes: Rita Levi Montalcini, Premio Nobel; Vittorio Feltri, director de L'Indipendente de Milan; Giorgio Grati, Presidente de la Pequeña Industria (Confindustria): Michele Placido, actor, etc.

${ }^{37}$ Per Un'ltalia Nuova, Roma, 1992, 7 pags.

${ }^{38}$ GINSBORG, op. cit., pag. 137.

${ }^{39}$ CORBETTA, PARISI y SCHADEI, op. cit., pp. 515-531.
} 
que no provoca crisis.

Programa. A favor de las reformas electorales e institucionales: elección directa del Jefe del Estado. Autonomía impositiva para regiones y ayuntamientos. En la lucha contra la mafia: defiende el empleo del Ejército en las zonas de mayor criminalidad y en casos de emergencia. Lema: "Elige un partido justo para un gobierno estable". Emblema: un sol naciente sobre el mar con las siglas del partido y la palabra "socialdemocracia", todo en rojo sobre fondo en blanco.

\section{Partido Liberal Italiano}

Nació en 1922 como heredero de los diversos partidos "liberales" que habían gobernado Italia desde su formación como Estado unificado ${ }^{40}$. La implantación del regimen fascista supuso la división del partido entre un sector dispuesto a colaborar con el nuevo régimen y un sector de oposición al mismo. En 1943 se procedió a la refundación del PLI (Croce, Einaudi). Desde entonces representa los intereses de los sectores con mayor poder económico del país. Durante el llamado período de reconstrucción (1945-51) el PLI ocupó los puestos claves de la política económica, el Banco de Italia y el Ministerio del Tesoro. En 1948 Luigi Einaudi fue nombrado Presidente de la República. En 1963 se opuso firmemente a la formación de los gobiernos de centro-izquierda. lo que le condujo a obtener el mejor resultado electoral de su historia (7\%) al recibir el apoyo de un sector de votantes de la $\mathrm{DC}^{41}$. En 1986 fue elegido Renato Altissimo secretario general del PLI. Desde la postguerra ha participado en la mayor parte de las mayorias gubernamentales. El partido tiene cuarenta mil miembros y forma parte del actual gobierno de coalición.

Programa. A favor de la reforma electoral en el sentido de adoptar el sistema francés: sistema mayoritario a doble vuelta. Elección directa del Jefe del Estado. Recursos impositivos para las regiones. Reduccion del déficit público y privatización de la RAI. Aumento de los jueces y policía para combatir a la mafia ${ }^{42}$. Lema: "No perdamos el tiempo. Darnos la fuerza para resolver los problemas". Emblema: una bandera italiana sobre la cual aparecen las siglas PLI.

\section{Movimiento Social Italiano}

Fundado en 1946 para reorganizar las fuerzas fascistas -en su versión social y republicana- tuvo siempre su principal base de apoyo en el Mezzogiorno ${ }^{43}$. En 1969 al moderado Michelini le sucede en la secretaria general Giorgio Almirante, el cual imprime un mayor dinamismo al partido, alternando la acción legal con la extralegal, hasta convertirlo, en las generales de 1972, en la cuarta fuerza política del

\footnotetext{
${ }^{40}$ Paolo FARNETT. II sistema dei partiti in Italia 1946-1979, il Mulino, Bologna, 1983, pag. 35 y 108-109.

${ }^{41}$ CORBETTA. PARISI y SCHADHE. op, cit., p. 183.

${ }^{42}$ La Repubblica, 24 de marzo de 1992.

${ }^{43}$ FARNETI, op. cit., pp. 41 y 112.
} 
país, con el $8,7 \%$ de los sufragios, su techo histórico ${ }^{44}$. Es la época en la que el MSI trata de convertirse en representante político de todo el área anticomunista, "la mayoría silenciosa", temerosa de la escalada terrorista de la extrema izquierda. En esa estrategia, en 1972, el partido se unifica con los monarquicos del PDIUM y pasa a denominarse Movimiento Social Italiano-Derecha Nacional (MSI-DN) ${ }^{45}$. Desde su nacimiento conviven en el partido dos tendencias, una más conservadora y otra radical. Su actual secretario general Gianfranco Fini, sucesor de Almirante en 1987, carece del carisma de su desaparecido líder. El partido cuenta con sesenta mil miembros. A efectos propagandísticos, consiguió incorporar a sus listas a la actriz de cine, Alessandra Mussolini, nieta del "Duce".

Programa. Mantenimiento del actual sistema electoral. Reforma institucional: establecimiento de la II República de carácter presidencialista y con representación parlamentaria de las categorías corporativas. Lucha eficaz contra la droga, la criminalidad y la mafia (incluyendo pena de muerte para los mafiosos), restaurar el orden público, aplicación en situaciones excepcionales de la ley marcial y del código militar. Replanteamiento de las fronteras nacionales históricas referidas a Istria, Fiume y Dalmacia. Impedir el ingreso en Italia de ciudadanos extracomunitarios durante un período de cinco años ${ }^{46}$. Lema: "Italia: necesidad de la Derecha", "Esta vez no tener miedo de tener corage. Vota y haz votar MSI-DN". Emblema: cartela con las siglas MSI y sobre ella la llama eterna tricolor.

\section{Liga Norte. Liga Lombarda}

Se funda en 1981 por Umberto Bossi. Consideran a la Lombardía como una nación que hunde sus raíces en el pasado histórico. En el año 1167 representantes de Milán, Cremona. Brescia, Bérgamo y Mantua, se reunieron en Pontida (Bérgamo) para sellar una alianza, llamada Liga Lombarda, con el fin de luchar por la autonomía contra el emperador Federico I "Barbarroja". Se presentan por vez primera en las elecciones administrativas de 1985 consiguiendo dos concejales en Varese ${ }^{47}$. En las generales de dos años después obtienen un diputado y un senador. A partir de 1989 comienza el ascenso espectacular de la .Liga Lombarda ${ }^{48}$. En diciembre de 1989 Bossi logra la formación de la Liga Norte, mediante la confederación de todas las Ligas existentes (Lombarda, Veneta, Piamonte, Liguria, Emilia Romagna y

${ }^{44}$ CORBETTA, PARISI y SCHADEE, op. cit., pp. 224-228. En las elecciones administrativas de 1971, el MSI obtuvo el $16,3 \%$ en Sicilia, convirtiendose en algunas ciudades en el partido mas votado, por ejemplo en Catania con el $21.9 \%$.

${ }^{45}$ Piero IGNAZI, Il Polo Esclisso. Profilo del Movimento Sociale Italiano, il Mulino, Bologna, 1989. pp. $148-165$.

46 Secolo d'ltalia, 1, 2 y 3 de abril de 1992.

47 Paolo NATALE, "I ega I omharda e insediamento territoriale: un analisi ecologica", en Renato MANNHEIMER, La Lega Lombarda, Jeltrinelli, Milano, 1991, pag. 85.

48 Ilvo DIAMANTI. La Lega. Geografia, storia e sociologia di un nuovo soggetto politico, Donzelli, Roma, 1993, pag. 19 y ss. 
Toscana) ${ }^{49}$. La más importante y la primera en nacer es la Liga Veneta, la "madre de todas las Ligas" ${ }^{\prime \prime}$. En la I Asamblea, celebrada en septiembre de 1990, aprueban defender una estructura federal para Italia que estaría formada por tres repúblicas -norte, centro y sur- ${ }^{51}$. Se presentan como un movimiento de protesta contra el sistema de partidos italianos, porque entienden que la actual partitocracia y la mafia están íntimamente relacionadas. Consideran que la actual política fiscal perjudica de forma objetiva a las regiones más ricas del país (el norte) en beneficio de un sur que no aprovecha estos recursos ajenos y los dilapida debido a su peculiar estructura política (partidos-mafia). Según su criterio, esta situación tiene un responsable: el gobierno de Roma. Se trata de un partido basado en un líder carismático, con grandes dotes de organizador ${ }^{52}$. Desde 1982 publican el semanario Lombardía Autonomista, que tiene actualmente una tirada de cincuenta mil ejemplares. Algunos comentaristas políticos de la prensa cotidiana, sin tiempo para la reflexión, se equivocan cuando lo definen como un partido fascista. Por el contrario, expertos politólogos, tras un análisis más ponderado lo califican como "un nuevo tipo de derecha"53, o "una forma de populismo antisistema"s4.

Programa: Autogobierno para Lombardía. Construcción de un estado federal con tres repúblicas. Defensa de la cultura, la historia y la lengua lombarda. Control del sistema financiero, de la administración pública, de la escuela, de las pensiones, etc, por parte del gobierno Lombardo. Los jóvenes deberán cumplir su servicio militar en Lombardia. Combatir con eficacia la delincuencia, el crimen y la mafia. Construcción de una Europa fundada en la autonomía y el federalismo ${ }^{55}$. Lema: "Roma ladrona, la Liga no perdona". "Contra los métodos mafiosos". Emblema: un guerrero medieval, con espada en alto y escudo, y en la parte superior el nombre del partido, todo ello en color azul sobre fondo blanco.

\section{Lista Pannella}

En esta ocasión el partido Radical no concurre a las elecciones y se presenta la "Lista Marco Pannella". nombre del líder histórico del partido Radical, que la encabeza ${ }^{56}$. Sus principales apoyos, además del propio partido Radical, son los pacifistas, federalistas, ecologistas y antiprohibicionistas.

Programa. Defienden una reforma electoral basada en el sistema mayoritario

${ }^{49}$ Jbidem, pag. 73.

${ }^{50}$ Ibídem, pp 43-54.

s1 Federalismo e Lega Lombarda, Brescia, 1991, 18 pags.

32 Umberto BOSSI y Daniele VIMISRCATI, Vento dal Nord, Sperling \& Kupfer, Milano, 1992.

${ }^{53}$ Gian Primo CELLA, "Leghe: s'ode a destra uno squillo di tromba", en I/ Bianco \& I/ Rasso, junio de 1990, pp. 6-8.

${ }^{54}$ Dwayne W(O)IS, "Les ligues régionales en Italie", en Revue Française de Science Politique, vol. $42, n^{\circ} 1$, février 1992, pag. 50.

"Sombardia Autonomiska, 11 de enero de 1992.

${ }^{56}$ La Repubblica. 4 de febrero de 1992. 
británico y norteamericano. Unificación de las diferentes fuerzas de orden público. Derecho al aborto más amplio. Menor limitación a la objección de conciencia. Desarrollo y consolidación del partido transnacional. Emblema: el nombre de Pannella con los signos de la paz y el antiprohibicionismo.

\section{Federación de los Verdes}

Después de muchas vicisitudes diversos grupos de Verdes formaron una federación. Tienen una dirección colegiada formada por once miembros representantes de los diferentes grupos y coordinados por el arquitecto Francesco Rutelli ${ }^{57}$. Estarían dispuestos a formar parte del gobierno, siempre que no fuera una reedición de la última coalición gubernamental.

Programa: Mantenimiento del sistema proporcional, con una barrera del $5 \%$. Reforzamiento del sistema parlamentario. Defensa de los derechos civiles. Reconversión ecológica de la producción, disminución de los gastos militares. lucha contra la contaminación atmosférica, reciclaje de los residuos sólidos. Prohibición de circulación automovilística privada en el centro de Milán, y sistema federal para la construcción europea. Lema: "Los otros te prometen la luna, nosotros te garantizamos la tierra". Emblema: el sol que ríe amarillo sobre fondo verde.

\section{Refundación Comunista}

Cuando la mayoría del histórico Partido Comunista Italiano decidió reconvertir su programa y cambiar su nombre -Partido Democrático de la Izquierda-, la minoría ortodoxa decidió constituir Refundación Comunista. Sus fundadores son Sergio Garavini, secretario general y Armando Cossutta, presidente, y tienen once diputados ex-PCI. En diciembre de 1991 celebraron en Roma su congreso constituyente, al que asistieron 1500 delegados en representación de sus ciento cincuenta mil militantes ${ }^{58}$. Apoyan a Refundación el vicjo grupo de "Il Manifesto" y el partido de la izquierda radical Democracia Proletaria, que en el 87 obtuvo ocho diputados.

Programa. A favor de mantener el actual sistema electoral proporcional. reducción del número de parlamentarios y oposición a la elección directa del Jefe de Estado. Redefinición de las industrias estatalizadas y lucha contra la evasión fiscal. En la batalla contra la mafia opuestos a la aplicacion de leyes especiales. Intensificar y ampliar la red de servicios sociales: sistema sanitario totalmente gratuito, reforma de las pensiones, jubilación a los 60 años, etc. Salida de Italia de la OTAN ${ }^{59}$. Lema: "El corazón de la oposición". Emblema: la clásica bandera roja con la hoz. el martillo y la estrella de cinco puntas.

\footnotetext{
${ }^{57}$ La Repubblica, 28 de marzo de 1992.

${ }^{58}$ La Repubblica, 13 de diciembre de 1991.

${ }^{59}$ Suplemento de Liberazione, 29 de fehrero de, 1992.
} 


\section{Rete: Movimiento por la Democracia}

Se trata de una nueva fuerza política fundada el 21 de marzo de 1991, por el ex-alcalde de Palermo. Leoluca Orlando. En los últimos tres años, Orlando se ha ganado amplias simpatías populares gracias a su decidida postura de lucha contra la mafia y a favor de la moralidad política. La Rete no es un partido sino "un conjunto de historias diversas para un fin común", según su fundador. El democristiano Leoluca Orlando, alcalde de Palermo, rompe en abril de 1989 con la coalición municipal y forma una nueva con democristianos, socialdemócratas, comunistas, verdes, e independientes católicos y de izquierda, dejando fuera a los socialistas. Su programa de gobierno se basaba en la lucha contra la mafia, la transpariencia en la administración y la solidaridad social. Boicoteado por su propio partido, su experiencia sólo duro ocho meses y en enero de 1990 se vió obligado a dimitir. Después de continuos enfrentamientos con la dirección democristiana decidió abandonar la DC en noviembre del mismo año y fundar un nuevo grupo político: Rete (Red).

Programa. La Rete considera muy grave la actual situación política e institucional y aboga por un cambio radical. Para ello es necesario restituir a la política dignidad moral y competencia para garantizar al sistema transpariencia y eficacia, combatir la maraña entre negocios y política, causa de la actual corrupción del sistema de partidos. Es preciso defender los valores de la justicia, la solidaridad y la democracia. Para ello es preciso abolir la inmunidad parlamentaria. Numerosos políticos, abogados y magistrados comprometidos, desde hace tiempo, en la lucha contra la corrupción y la mafia, se presentan como candidatos de la Rete: Nando Dalla Chiesa, hijo del famoso general Dalla Chiesa asesinado por la mafia; Alfredo Galasso, abogado en la parte civil en el maxiproceso a la mafia y en el proceso Ustica; Diego Novelli, ex-alcalde comunista de Turín, etc ${ }^{60}$. Lema: "La Rete sois vosotros". Emblema: la silueta de varias personas formando una red.

\section{Otros partidos}

Además de los partidos tradicionales y de los de reciente fundación, concurren también otros, algunos con pintorescas denominaciones. Así, el Partido de Caza, Pesca y Ambiente, dice representar los intereses de los 400.000 cazadores italianos y tiene 135 concejales en diversos Ayuntamientos. El Partido de los Pensionistas, fundado en 1987, tiene diez mil miembros sobre todo en el norte del país. La lista Federalismo, Pensionistas y Hombres Vivos, que incluye al Movimiento Sardo y a la Unión del Valle de Aosta. El Partido del Amor, que intenta recoger la herencia de la famosa Ilona Staller "Cicciolina", diputada en la anterior legislatura y nuevamente candidata junto a la también porno-star Moana Pozzi.

\footnotetext{
${ }^{60}$ Panorama, 5 de abril de 1992, pag. 81.
} 


\section{Grupos transversales}

Una particularidad de la actual política italiana son los llamados grupos transversales. De todos son conocidas, las múltiples dificultades del sistema político, situación que lleva a algunos politólogos a hablar de la crisis de la democracia de los partidos $^{61}$. Por ello, algunos grupos de personalidades -militantes de diversos partidos políticos- han tomado la iniciativa de organizar movimientos que se comprometan a llevar a cabo reformas en el sistema político. Estos grupos basan sus programas en una imprescindible moralización de la vida pública y de las costumbres políticas mediante reformas institucionales pactadas. Se trata de un fenómeno en aumento si bien sólo implica a las élites políticas ${ }^{62}$. El ejemplo más importante es el llamado Pacto por la Reforma que agrupa a 450 candidatos a la Cámara procedentes de distintos partidos -DC, PSI, PDS, PRI, PLI, PSDI- ${ }^{63}$, y de los cuales resultaron elegidos 110 .

\section{LA CAMPAÑA ELECTORAL}

Varias son las características de la campaña electoral italiana. La publicidad se desarrolla básicamente en la prensa y en la televisión y no en la calle. Destaca la escasez de carteles en las vías públicas -además, sólo se colocan en los lugares acondicionados expresamente para ello- y la total ausencia de vallas publicitarias, lo que proporciona un tono de frialdad a la campaña, si la comparamos con las españolas. La publicidad se centra en los medios de comunicación: prensa, radio y sobre todo televisión. Esta situación lleva a que la lucha por el control de los canales públicos televisivos se intensifique en este período. Por ejemplo, la suspensión del famoso programa de debate político "Samarcanda" de la "RaiTre", por parte del Gobierno $^{64}$. Por otra parte. los espacios electorales televisivos de carácter gratuito cedidos a los partidos, tienen la misma duración -tres minutos- para todos. Con lo que se garantiza la igualdad de oportunidades para todas las formaciones políticas, con total independencia de sus resultados anteriores ${ }^{65}$.

Uno de los principales temas tratados fue las distintas posibilidades de alianzas gubernamentales, imprescindibles para poder realizar las reformas ofrecidas en los programas, pasando estos a segundo plano. Durante la campaña, los partidos aprovecharon diversas situaciones concretas para incorporarlas a ella: el asesinato del

${ }^{61}$ SCOPPOL.A, op. cit., pp. 424-437.

${ }^{62}$ SANI, op. cit., pag. 135.

${ }^{63}$ La Repubblica, 6 y 18 de marzo de 1992.

${ }^{64}$ La Repubblica, 20 de marro de 1992.

${ }^{65}$ Sería necesario resaltar la gran diferencia de las campañas italianas con las desarrolladas en nuestro país. Por ejemplo, frente al injusto reparto del tiempo televisivo y al derroche publicitario de los partidos españoles contrasta la igualdad de tiempo para todas las fuerzas politicas en las televisiones públicas y la casi austeridad de los partidos italianos. 
eurodiputado de la DC. Salvo Lima ${ }^{66}$, o el rumor sobre un supuesto plan desestabilizador del sistema democrático ${ }^{67}$.

La campaña de la DC se basó en los logros conseguidos por sus anteriores gobiernos, en el peligro que significaría una situación política inestable, jugó con el miedo a una fragmentación del país y apoyó la necesidad de la convergencia con Europa, para la cual era precisa una "Italia fuerte y unida". Propuso a sus actuales aliados una nueva edición de la coalición de cuatro partidos (DC-PLI-PSDI-PSI), bajo el liderazgo de la $\mathrm{DC}^{\mathbf{8} 8}$.

El PSI realizó una campaña basada en su líder Craxi, presentándose como alternativa para encabezar un futuro gobierno fuerte de coalición que garantizase la estabilidad. Otra de sus estrategias fue intentar convertirse en el primer partido de la izquierda, desplazando al PDS de su actual primacía ${ }^{69}$.

El PDS centró su campaña sobre la posibilidad de su entrada en un gobierno de amplia coalición, en el que por encima de las personas primasen los programas. Considera necesario construir una izquierda fuerte (para lo cual intenta atraerse al PSI) para poner fin a los cuarenta años de gobierno de la DC. Por otra parte, el peligro de una erosión de votos por su izquierda -debido a la presencia de Refundación- le llevó a recordar a los electores sus raíces comunistas ${ }^{70}$.

Uno de los temas más sugestivos de la campaña desarrollada por el PRI, fue la propuesta de La Malfa sobre la formación de un "gobierno de técnicos" cuya misión sería realizar las reformas imprescindibles de las instituciones, tras lo cual se disolvería ${ }^{71}$. Esta propuesta significa el reconocimiento del fracaso de los políticos tradicionales en depurar la política italiana. Además La Malfa anunció que no pactaría ni con la DC ni con el PSI. un nuevo gobierno igual a los anteriores ${ }^{72}$.

La Liga Norte-Liga Lombarda realizó una campaña relativamente más moderada que la anterior del otoño del 91 en las municipales de Brescia ${ }^{73}$, cuando alcanzó su primer gran éxito ${ }^{74}$. Posiblemente debido a la influencia de la figura ascendente de su ideólogo. el profesor Miglio. Centraron su campaña en un fuerte ataque al Gobierno de Roma, que genera una política impositiva por la cual -según ellos- toda la riqueza creada por el Nortc, es dilapidada en el Sur. También fue blanco de sus ataques la actual partitocracia y sus vinculaciones con la mafia. Asímismo destacaron

${ }^{66}$ La Repubblica. 14 y 15 de marzo de 1992.

${ }^{67}$ La Repubblica, 20 de marzo de 1992.

${ }^{68}$ Il Popolo, 1,2,3 y 4 de abril de 1992.

${ }^{69}$ Avanii !, 24 y 28 de marzo y $1.2,3$ y 4 de abril de 1992.

${ }^{70}$ L'Unità. 1.2.3.4 y 5 de abril de 1992.

"La Repubblica, 26 de marzo de 1992. La idea no es nueva, en 1981. Bruno Vicentini, lider republicano había presentado una propuesta análoga. Se equivoca el profesor Ramon Cotarelo cuando identifica a La Malfa con el pensamiento fascista, véase Diario 16, 8 de abril de 1992, p. 5.

${ }^{72}$ La Voce Repubblicana, 23-24 de marzo de 1992.

${ }^{73}$ Lombardia Autonomista. 13 de noviembre de 1991.

${ }^{74}$ L'Indipendente, 26 de noviembre de 1991 . Los resultados fueron los siguientes: Liga: $24.4 \%$; DC: 24,3\%: PSI: 10,3\%: PIS: 9.4\%: PRI: 5,5\%; etc. El porcentaje de votantes fue del $89.2 \%$ del censo. 
su vocación federalista ${ }^{75}$.

La campaña de la Rete fue modesta comparada con las de los grandes partidos, por la falta de medios económicos. Toda ella se basó en la idea de extender la moralidad y la ética a la política. Para Orlando "es necesario imponer la honestidad como valor político". Se acusó a la DC de carecer de ella y se atacó directamente al presidente del Consejo. "El enemigo es Andreotti" señaló su fundador ${ }^{76}$.

\section{LOS RESULTADOS}

Los resultados totales del Congreso de los Diputados, junto a los de las elecciones de 1987, aparecen en el cuadro $n^{\circ} 1$. La participación electoral disminuyó ligeramente respecto a la anterior consulta. De los 47.431 .482 electores inscritos en el Censo Electoral votaron el $87,2 \%$ (en 1987: $88,8 \%$ de votantes) ${ }^{77}$. No obstante tenemos que recordar que Italia es uno de los paises europeos con mayor índice de participación electoral ${ }^{78}$.

Tres fueron las ideas destacadas por los más importantes diarios italianos, tras conocerse los resultados finales: las elecciones habían sido un "terremoto" para el sistema político, el voto de castigo recibido por los partidos históricos y el derrumbe democristiano ${ }^{79}$. Efectivamente, todos los grandes partidos sufren unos resultados adversos a sus espectativas. La Democracia Cristiana encaja una derrota histórica, al caer por primera vez -desde 1948- por debajo del $30 \%$ y pierde casi un $5 \%$ con respecto a las legislativas del 87. El Partido Democrático de la Izquierda, padece una doble derrota: por una parte, disminuye un $10,5 \%$ con respecto a los resultados del $\mathrm{PCI}$ en los anteriores comicios, y por otra, la mitad de ellos se dirigen a Refundación Comunista (que obtiene un buen resultado). No obstante, el PDS continúa siendo la segunda fuerza del país, y la primera de la izquierda (objetivo primordial de su estrategia política). El Partido Socialista, soporta también una derrota, no tanto por la pérdida porcentual de un $0.7 \%$, sino porque no logra situarse como primera fuerza de la izquierda, meta expresada durante toda la campaña electoral. Los partidos

${ }^{75}$ Lombardia Autonomista, 14. 21 y 28 de marzo de 1992.

${ }^{76}$ La Repubblica, 29 de marzo de 1992.

${ }^{77}$ La Repubblica, 4 y 7 de abril de 1992. Para cubrir los 630 escaños de la Cámara concurrieron 8.860 candidatos. De los 247 símbolos presentados fueron admitidos 117 .

${ }^{78}$ En el periodo 1945-1980, la participación electoral media se situo en el $92,6 \%$ de los inscritos en el Censo Electoral. Renato MAN NHIEIMISR y Giacomo SANI, II mercato elettorale. Identikit dell' elettore italiano, il Mulino, Bologna, 1987, pag. 33 y 46.

${ }^{79}$ Veamos los titulares, de algunos importantes diarios, correspondientes al dia 8 de abril. La Stampa (Turin): "Elecciones, un terremoto": Corriere della Sera (Milan): "Italia protesta, elecciones terremoto": La Repubblica (Roma): "Se ha derrumbado el muro DC. El voto de protesta ha castigado también a Craxi. El Gobierno está en minoría": /l Messaggero (Roma): "ltalia sin mayoria. Resultado a la polaca: crece el voto de protesta y la fragmentación parlamentaria". Y los diarios de partidos: Il Popolo (DC'): "Gobernabilidad en riesgo": Avanti! (PSI): "Un terremoto politico"; L'Unità (PDS): "Se acabó el régimen democristiano". 
centristas se mantienen, destacando la subida de $0,7 \%$ del PRI, beneficio conseguido gracias a su salida del gobierno. La extrema derecha, representada por el MSI-DN, sufre una pequeña pérdida de votos. Entre las nuevas formaciones políticas, destaca el casi $2 \%$ conseguido por la Rete -éxito personal de Orlando-, pues se trata de papeletas inequívocamente anticorrupción. Finalmente, tenemos que señalar el impresionante ascenso de la Liga Norte, que pasa del $0,7 \%$ al $8,7 \%$-de 1 diputado a 55- convirtiéndose en la primera fuerza política en varias importantes ciudades del norte -Milán, Mantova, Pavia. Como o Varese- y en la cuarta a escala nacional. Umberto Bossi, su máximo dirigente, fue el líder más votado en todo el país: en la circunscripción de Milán-Pavia obtuvo 240.513 votos $^{80}$. Es interesante constatar la procedencia del voto legista y sus zonas de influencia. Mientras en la "zona roja" del valle del Po (de hegemonia comunista) avanzó un 10\%, en la "zona blanca" en torno a Venecia (antiguo feudo democristiano), dobló la cifra anterior ${ }^{81}$.

Si analizamos los resultados a nivel nacional se observa un país dividido en tres grandes zonas. Al norte. la "Italia leghista" (Lombardia, Piamonte, Liguria, Veneto, Friuli); en el centro la "Italia roja" (Emilia Romagna, Toscana, Umbria) con predominio del PDS y Refundación; y al sur la "Italia tradicional" (todo el sur y las islas) con hegemonía de la $\mathrm{DC}^{82}$.

De los resultados se desprende la pérdida de la mayoría absoluta de la coalición de cuatro partidos gobernante (DC+PLI+PSDI+PSI), lo que conduce a una mayor inestabilidad, pero al mismo tiempo abre, en principio, nuevas espectativas en la configuración de un nuevo bloque de gobierno -con la posibilidad de la incorporación de fuerzas que hasta el presente habían estado marginadas-, precisamente en una legislatura que tendrá que abordar profundas reformas institucionales, a tenor de las propuestas electorales de los principales partidos.

Para concluir debemos señalar un importante fenómeno producido en estas elecciones: la redistribución territorial de las bases tradicionales de apoyo partidista. El travase de votos hacia la Liga Norte, provoca una meridionalización de la política italiana, sobre todo en la DC y en el PSI. Es decir, que mientras en el norte. la Liga se convierte en partido ascendente. desplazado a la DC y al PSI, ahora, la base fundamental de los votantes de estos dos partidos se localiza en el sur.

\footnotetext{
${ }^{80}$ La Repubblica y Corriere della Sera, 8 de abril de 1992.

${ }^{81}$ Mark GILBERT. "Warriors of the new Pontida: the challenge of the Lega Nord to the italian party system", en The Political Quarterly, volumen 64, $n^{2} 1$, enero-marzo 1993, pag. 103.

${ }^{82}$ Gianni STATHRA. Come votano gli italiani. Sperling \& Kupfer. Milano, 1993, pag. 105.
} 


\section{CUADRO I}

Resultados totales de las elecciones generales de 1987 y 1992 en Italia (Cámara de Diputados)

\begin{tabular}{||l|c|c|c|c|c|c||}
\hline \multirow{3}{*}{ PARTIDOS } & \multicolumn{3}{|c|}{1992} & \multicolumn{3}{|c||}{1987} \\
\cline { 2 - 7 } & $\%$ & votos & escaños & $\%$ & votos & escaños \\
\cline { 2 - 7 } & & & & & \\
\hline DC & 29.7 & 11.627 .657 & 206 & 34,3 & 13.233 .620 & 234 \\
PDS & 16.1 & 6.315 .815 & 107 & $26.6^{1}$ & 10.250 .644 & 177 \\
PSI & 13,6 & 5.336 .358 & 92 & 14,3 & 5.501 .696 & 94 \\
LIGA & 8.7 & 3.394 .917 & 55 & 0,7 & 258.319 & 1 \\
REF & 5,6 & 2.202 .574 & 35 & $1,7^{2}$ & 641.901 & 8 \\
MSI & 5.4 & 2.103 .692 & 34 & 5.9 & 2.281 .126 & 35 \\
PRI & 4.4 & 1.721 .658 & 27 & 3.7 & 1.428 .663 & 21 \\
PLI & 2,8 & 1.119 .492 & 17 & 2,1 & 809.946 & 11 \\
Verdes & 2,8 & 1.092 .783 & 16 & 2,5 & 969.218 & 13 \\
PSDI & 2.7 & 1.063 .048 & 16 & 2,9 & 1.140 .209 & 17 \\
RETE & 1,9 & 728.661 & 12 & - & - & - \\
Panella & 1,2 & 485.339 & 7 & $2,6^{3}$ & 987.720 & 13 \\
Federal. & 0.4 & 154.510 & 1 & 0.5 & 211.685 & 3 \\
Referen. & 0.8 & 319.327 & - & - & - & - \\
Pension. & 0.6 & 220.740 & - & 0.8 & 298.402 & - \\
Otros & 3.3 & 1.322 .416 & 5 & 1,4 & 558.352 & 3 \\
\hline
\end{tabular}
(1) Entonces $\mathrm{PCl}$
(2) Entonces DP
(3) Entonces PR 